$\stackrel{N / N}{=}$

Global burnals Inc.

औf

GLOBAL JOURNAL OF MEDICAL RESEARCH: C

MICROBIOLOGY AND PATHOLOGY

Volume 20 Issue 5 Version 1.0 Year 2020

Type: Double Blind Peer Reviewed International Research Journal

Publisher: Global Journals

Online ISSN: 2249-4618 \& Print ISSN: 0975-5888

\title{
Immune Response after Three Doses of Hepatitis B Vaccine among Children below Five Years of Age in Mwanza, Tanzania
}

By Delfina R. Msanga, Raphael Rwezaula, Semvua Kilonzo, Elizabeth Kwiyolecha, Tulla Masoza, Emmanuel Mkumbo, Mariam M. Mirambo \& Stephen E. Mshana

Catholic University of Health and Allied Sciences

Abstract- Background: Hepatitis B virus (HBV) infections is moderately endemic in many countries in the sub-Saharan Africa including Tanzania. Immunization of children below five years of age has been found to be an effective strategy in controlling infectious diseases. However, the data regarding immune responses following vaccination are very limited in low-income countries. Here, we report the sero-conversion among children below five years of age after three doses of HBV vaccine in Mwanza, Tanzania.

Methodology: A cross-sectional study involving children below five years of age was conducted at Makongoro Reproductive and Child Health $(\mathrm{RCH})$ clinic between May and June 2017. Sociodemographic data were collected, and vaccination status was confirmed from reproductive and child health $(\mathrm{RCH})$ cards. Serum HBV surface antibodies (anti-HBs) were quantified using enzyme immunoassay (Enzygnost Anti-HBs II). Data were analysed by using STATA version 13 software.

Keywords: hepatitis B, children, immune response, seroconversion, mwanza, Tanzania.

GJMR-C Classification: NLMC Code: WO 680

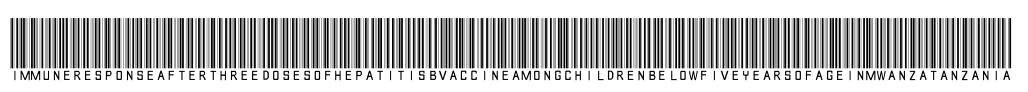

Strictly as per the compliance and regulations of:

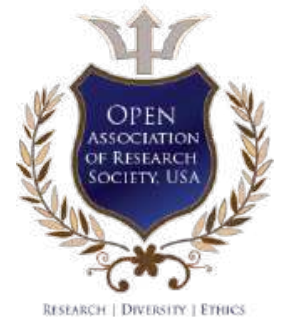

(C) 2020. Delfina R. Msanga, Raphael Rwezaula, Semvua Kilonzo, Elizabeth Kwiyolecha, Tulla Masoza, Emmanuel Mkumbo, Mariam M. Mirambo \& Stephen E. Mshana. This is a research/review paper, distributed under the terms of the Creative Commons Attribution-Noncommercial 3.0 Unported License http://creativecommons.org/licenses/by-nc/3.0/), permitting all non-commercial use, distribution, and reproduction in any medium, provided the original work is properly cited. 


\title{
Immune Response after Three Doses of Hepatitis B Vaccine among Children below Five Years of Age in Mwanza, Tanzania
}

\author{
Delfina R. Msanga ${ }^{\alpha}$, Raphael Rwezaula $^{\circ}$, Semvua Kilonzo $^{\circ}$, Elizabeth Kwiyolecha $^{\omega}$, Tulla Masoza $^{*}$, \\ Emmanuel Mkumbo ${ }^{\S}$, Mariam M. Mirambo ${ }^{\star} \&$ Stephen E. Mshanav
}

\begin{abstract}
Background: Hepatitis B virus (HBV) infections is moderately endemic in many countries in the sub-Saharan Africa including Tanzania. Immunization of children below five years of age has been found to be an effective strategy in controlling infectious diseases. However, the data regarding immune responses following vaccination are very limited in low-income countries. Here, we report the sero-conversion among children below five years of age after three doses of HBV vaccine in Mwanza, Tanzania.
\end{abstract}

Methodology: A cross-sectional study involving children below five years of age was conducted at Makongoro Reproductive and Child Health (RCH) clinic between May and June 2017. Socio-demographic data were collected, and vaccination status was confirmed from reproductive and child health $(\mathrm{RCH})$ cards. Serum HBV surface antibodies (anti-HBs) were quantified using enzyme immunoassay (Enzygnost Anti-HBs II). Data were analysed by using STATA version 13 software.

Results: A total of 300 children were enrolled with the median age of 15 (Interquartile range [IQR]: 9-22.5) months. The median interval from last dose to the time of evaluation was 10(IQR: 5-18) months with all children assessed8weeks post-vaccination. Out of 300 children, 265(88.3\%, 95\% Confidence interval $[\mathrm{Cl}]: 84-91$ ) had $\geq 10 \mathrm{lU} / \mathrm{L}$ of anti $-\mathrm{HBs}$ indicating sero-conversion. The median titres among those who seroconverted was 66(IQR: 26-134) IU/L. The median age at the time of evaluation of those who seroconverted was significantly lower than those who were not seroconverted [14(IQR: 9-21) vs. 18(IQR: 11-26, $P=0.03$ ]. The titres were found to decrease significantly by 0.84 and $0.98 \mathrm{IU} / \mathrm{L}$ with a unit increase in interval from last dose and a unit increase in age, respectively.

Conclusion: There is high seroconversion after three doses of HBV vaccine among children in Tanzania which is associated with young age. Further studies to evaluate immunogenicity of HBV vaccine in different age groups are recommended in resource limited countries to provide data that regarding booster dose recommendation. In addition, there is a need to evaluate immunization efficiency by determining immunogenicity of one of the vaccine component in the package as a proxy indicator.

Author $\alpha \sigma \omega ¥:$ Department of Pediatrics and Child health, Weill Bugando School of Medicine, Catholic University of Health and Allied Sciences, P.O. Box 1464, Mwanza, Tanzania.

Author $\& \quad v \quad x$ : Department of Microbiology and Immunology, Weill Bugando School of Medicine, Catholic University of Health and Allied Sciences, P.O. Box 1464, Mwanza, Tanzania.

e-mail:mmmirambo@gmail.com

Author p: Department of Internal Medicine, Weill Bugando School of Medicine, Catholic University of Health and Allied Sciences, P.O. Box 1464, Mwanza, Tanzania.
Keywords: hepatitis $B$, children, immune response, seroconversion, mwanza, Tanzania.

\section{BACKGROUNDS}

(n) epatitis B virus (HBV) infection is one of the most common diseases across the globe with one third of the population estimated to be infected[1]. About $5 \%$ of total world population are chronic carriers and nearly a quarter of these carriers develop liver cirrhosis and hepatocellular carcinoma[2] with about one million deaths being reported annually [3]. Therapeutic options for treating HBV chronic infections are difficult to implement and are not yet fully effective in many settings particularly in resource limited countries. Vaccination remain to be an effective measure to prevent HBV infections. Effective vaccination has been found to reduce HBV infections, therefore reducing the risk of transmission to the susceptible contacts[4]. In the intermediate and high endemic regions, individuals are at high risk of acquiring HBV infection if vaccination is not implemented [1]. The World Health Organization (WHO) recommends that HBV vaccination should be part of national immunization programs for countries with HBV carrier prevalence of $8 \%$ or greater, to reach a goal of reducing a proportion of chronic carriers and complications associated with HBV infections [5, 6].

In Tanzania the inclusion of HBV vaccine in childhood immunization program was first implemented in 2013 and the vaccine is administered $0.5 \mathrm{ml}$ intramuscular on fatty tissue over anterolateral thigh muscle at weeks 6,10 and 14 respectively after birth in combination with other 4 vaccines in the package (pertussis, diphtheriae, tetanus and Haemophilus influenza type B). It is estimated that this standard schedule of immunization should produce about 95\% seroprotection [6]. Despite the reported high seroconversion following HBV vaccination in other countries, there are variations in these proportions among different geographical areas with different endemicity status[7]. A previous study[8] in Dar es Salaam among children below five years of age reported sero-conversion of $69 \%$. 
Different factors including storage conditions, different forms of immunosuppression, genetic makeup etc. have been implicated to affect the immune response to HBV vaccination[9, 10]. In the countries like Tanzania, where there is no routine assessment of immune response which will lead to additional dose for non-responders, there is a need of data to evaluate DPT-HBV programme after 6 years of its implementation. In addition, there is limited data on the efficiency of childhood immunization particularly in vaccines which are given in combinations in Tanzania. Some previous studies evaluated efficiency of childhood immunization by using other components such as diphtheriae and tetanus toxoid (TT) vaccine [11-13] while others used pertussis component. In a view of that, the study was designed to assess the immunogenicity of HBV vaccine among children who completed three doses, the information that may be useful in controlling vaccine preventable diseases in Tanzania.

\section{Methods}

a) Study design, study area and study population

The cross-sectional study was conducted from May to June 2017 among children under five years of age from Makongoro reproductive and child health $(\mathrm{RCH})$ clinic. This facility had no any report of cold chain problems in routine assessment.

\section{b) Sampling and inclusion criteria}

The sample size was calculated using Kish Lisle formula using the prevalence of $87 \%[14]$. Children under five years of age who had received three doses of HBV vaccine (Pentavalent Vaccine-DPT-HepB-Hib) were serially enrolled until the sample size was reached. The study included only children who had received three doses of Pentavalent Vaccine (DPT-HepB-Hib) with the last dose given at least 8 weeks ago. To avoid nonresponders due to chronic HBV infection, all children who were HBsAg positive were excluded from the study.

\section{c) Laboratory procedures}

About $3 \mathrm{ml}$ of venous blood was aseptically collected using plain vacutainer tubes (BD, Kenya, and Nairobi) and transported to BMC accredited laboratory for processing. The anti-HBs titres were quantified using enzyme immunoassay (SIEMENS, Enzygnost ${ }^{\circledR}$ Anti-HBs II, and Germany) following manufacturer's instructions to detect the presence of specific anti-HBs. The presence of anti-HBs greater than $10 \mathrm{IU} / \mathrm{L}$ was defined as presence of protective antibodies.

\section{d) Data management and analysis}

Data were entered and analysed using a STATA version 13. Continuous variables were summarized as median with inter-quartile range and categorical variables were summarized as proportions. Rank sumMann Whitney test was used to compare the median titres, weight, age and interval from the last dose. Using immune response as outcome, multivariate logistic regression analysis was done. However, weight and interval were not included in the model because of their collinearity with age. In all children with titres greater than $10 \mathrm{IU} / \mathrm{L}$, regression analysis was done to determine the correlation between age, interval from the last dose and titers. A predictor with a $P$ value of $<0.05$ was considered statistically significant.

\section{Results}

a) Socio-demographic characteristics of the enrolled children

A total of 300 children under five years of age who received three doses of HBV vaccine were enrolled. There was almost equal distribution between females (49\%) and males (51\%). The median age of enrolled children was 15 (Interquartile range [IQR]: 9-22.5) months. The median interval from last dose to the time of evaluation was 10(IQR: 5-18) months with all children assessed $\geq 8$ weeks post -vaccination. The median duration for breastfeeding was 12(IQR:9-15.5) months (Table1). All children had no co-morbidities.

Table 1: Characteristics of enrolled children and seroconversion

\begin{tabular}{|l|c|c|c|c|c|}
\hline \multicolumn{1}{|c|}{ Variable } & \multicolumn{3}{c|}{ Univariate analysis } & \multicolumn{2}{c|}{ Multivariate } \\
\hline & Positive & Negative & P-value & OR[95\%Cl] & P-value \\
\hline Age(months) & $14($ IQR:9-21) & $18($ IQR:11-36) & 0.030 & $0.96(0.94-0.99)$ & 0.005 \\
\hline Breastfeeding duration (months) & $12($ IQR:9-16) & $12(9-14)$ & 0.766 & $1.29(0.59-2.79)$ & 0.514 \\
\hline Interval from the last dose(months) & $10($ IQR:5-17) & $13($ IQR:5-31) & 0.103 & & \\
\hline Weight(kgs) & $9.5(\mathrm{IQR}: 8.4-11.2)$ & $10.4(\mathrm{IQR}: 9-13.5)$ & 0.014 & & \\
\hline Sex & & & & & \\
\hline Female & $127(86.4)$ & $20(13.6)$ & & & \\
\hline Male & $138(90.2)$ & $15(9.8)$ & 0.305 & $1.39(0.67-2.87)$ & 0.370 \\
\hline
\end{tabular}

b) Seroconversion and associated factors

Out 300 children, 265(88.3\%, 95\% Confidence interval $[\mathrm{Cl}]: 84-91)$ had $\geq 10 \mathrm{IU} / \mathrm{L}$ anti $-\mathrm{HBs}$ indicating sero-conversion. The median titres among those who seroconverted was 66(IQR: 26-134) IU/L. The median age at the time of evaluation of those who seroconverted was significantly lower than those who were not seroconverted [14(IQR: 9-21) vs. 18(IQR: 1126, $\mathrm{P}=0.03$ ] (Figure1). 


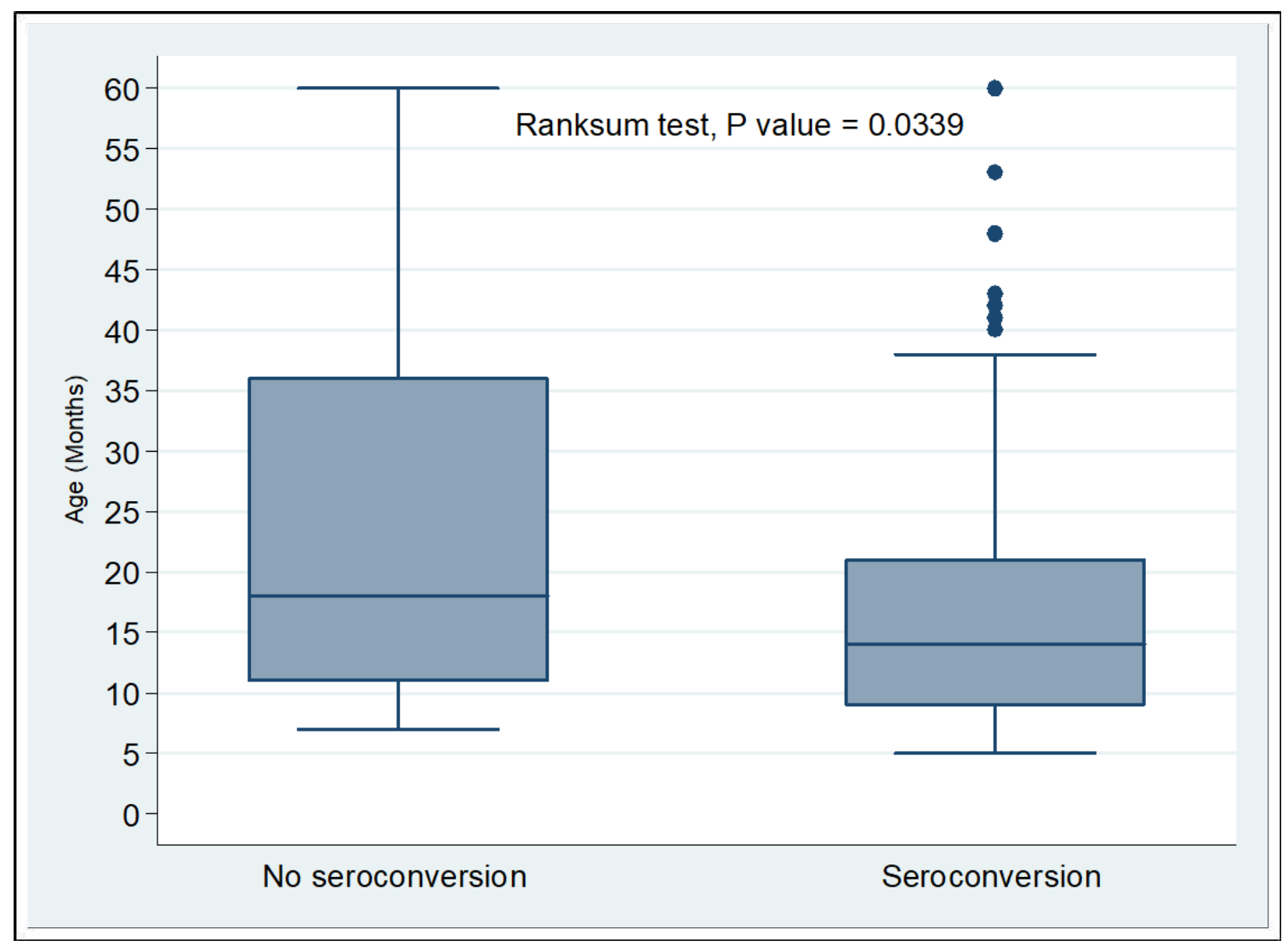

Figure 1: Box plot showing median age of those with seroconversion and those with no seroconversion

By multivariate logistic regression analysis decrease in age was independently associated with seroconversion (OR: 0.96(95\%Cl: 0.94-0.99, $P=.005)$. The titres were found to decrease significantly as the age increases $($ Coef $=-0.96,95 \% \mathrm{Cl}-1.700--0.274, P=.007)$ (Figure2).

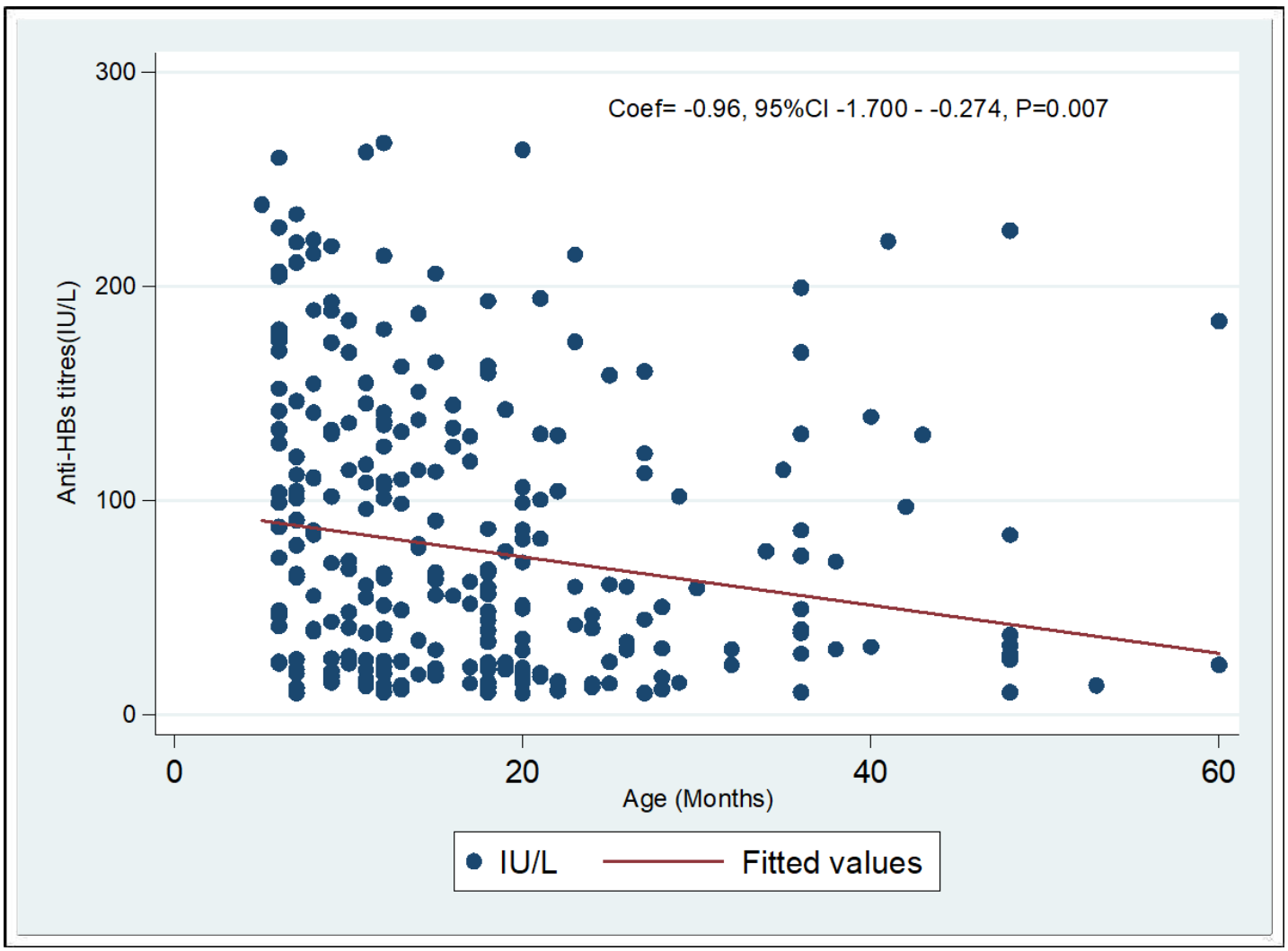

Figure 2: Scatter diagram with fitted line showing correlation between age and anti-HBs titres 
In addition, the titers were also found to decrease as the interval from the last dose increases (Coef $=-0.84$, $95 \% \mathrm{Cl},-1.591--0.106, P=.025)$ (Figure3).

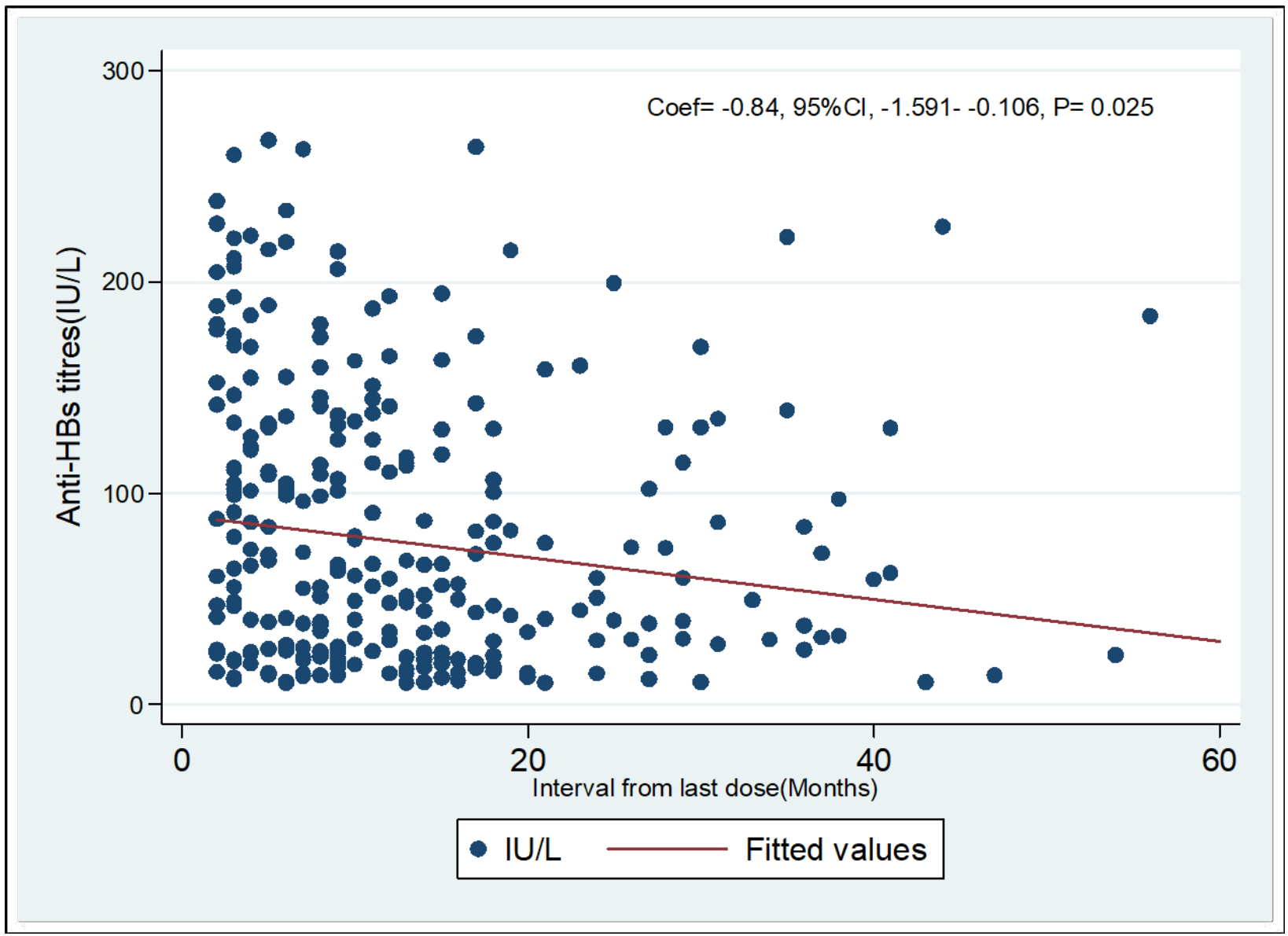

Figure 3: Scatter diagram with fitted line showing correlation between interval from last dose and anti-HBs titres

\section{Discussion}

One of the key aspects in vaccination programs in resource constrained countries is to ensure potency of the vaccine by maintaining the cold chain. Therefore, there is a need for regular studies to assess immunogenicity of vaccines especially those which are given in combination to provide a proxy indicator for the efficiency of other vaccines in the package. In Tanzania, HBV vaccine is given in combination with Corynebacterium diphtheriae, Bordetella pertussis (whooping cough), Clostridium tetani (tetanus) and Haemophilus influenza type B (influenza). However, there is paucity of data on immunogenicity of these vaccines.

To the best our knowledge, this is the first study to assess immune response after HVB vaccine among children in Mwanza, Tanzania. In the present study about $90 \%$ of children seroconverted after three doses of HBV vaccine. The observed high seroconversion and sustained high HBV vaccination coverage of 92.5\% [15] reported in Mwanza will eventually reduce the transmission of HBV in future. In addition, this information can be used as a proxy indicator for efficiency of other vaccines given in combination with
HBV vaccine in Tanzania. The reported seroconversion rate in the current study is consistent with the previous reports which documented the seroconversion of $87 \%$, $81.5 \%, 94.1$ and $96.7 \%$ [14, 16-19]. In the contrary, the reported seroconversion in the current study is higher than reported previously in Dar es Salaam and other endemic areas[8, 20]. Variations in seroconversion might be attributable to the type of vaccine used in terms of synthesis and preparations etc; in the current study the vaccine used was Pentavalent Vaccine (DPTHepB-Hib) which might be different from other studies where monovalent HBV was used [14, 16]. In addition, amount of antigen delivered, genetic variation among the population involved, vaccination coverage, endemicity status, faults in vaccine cold chain, methods used to evaluate antibody titers etc. might contribute to the observed discrepancies [21-24]. Moreover, in this study, about $11.7 \%$ of children were found to be nonresponders after receiving three doses of HBV vaccine which is slightly lower than $14.6 \%$ and $15.6 \%$ reported in previous studies[25, 26]. The possible explanation could be genetic variability and impaired lymphocyte activation as reported earlier [19, 27-29]. It should be noted that children studied in the current study were healthy with 
no co-morbidities. Genetic factors and primary immunodeficiencies could were not ruled.

In the current study, it was observed that, as the age increases by one month, the anti-HBs titers were found to decrease by $0.96 \mathrm{IU} / \mathrm{L}$. It was further observed that, the anti-HBs titers decrease by $0.84 \mathrm{IU} / \mathrm{L}$ as the interval from the last dose increases by one month. With this trend, by the age of 10 years most of these children would have undetectable levels of anti-HBs titers necessitating the need for considering booster dose to provide long lasting protection. Cohorts with long term follow-ups are recommended in this setting to evaluate the need for a booster dose. This observation is consistent to what was reported earlier [16, 20, 30-32] whereby the anti-HBs titers were found to decrease as the age increases and almost undetectable to a significant proportion of children by the age of 11 years. In the contrary some other studies concluded that, there is no need for booster dose after receiving 3-dose schedule of HBV vaccine since the anti-HBs titers can persist for longer period [33] while another study confirmed that there is long lasting cellular immunity despite decrease anti-HBs levels[34]. This conflicting information could be due to endemicity status in the study areas. Further studies to evaluate the levels of anti-HBs titers and cellular immunity among different age groups are highly recommended in areas with different endemicity status.

Regarding sex, in the current study, there was no significant difference in the levels of anti-HBs titers among female and male children which is similar to the previous reports $[14,25,35]$. This could be explained by the fact that there was almost equal distribution between males and females with equal distribution of factors that could influence seroconversion and level of titres.

Limitations of this study include: Inability to assess other forms of primary immunodeficiencies and genetic conditions which might impair immune response to vaccines and contributes to a significant proportion of non-responders and failure to give birth dose as it is not included in Tanzania Immunisation Vaccination and Development Program.

\section{Conclusion}

There is high seroconversion after three doses of HBV vaccine among children in Mwanza city which is associated with young age. Further studies to evaluate the level of protective antibodies at different age groups are recommended across the country and other resource constrained countries. This necessary especially in deciding the issue of dose at birth and booster dose in ration to HBV vaccination. High seroconversion of HBV vaccine signifies the effectiveness of other childhood vaccines in Tanzania.

\section{List of abbreviation}

Anti-HBs: Antibodies against hepatitis B surface antigen CUHAS: Catholic University of Health and Allied Sciences

Cl: Confidence interval

ELISA: Enzyme-linked immunosorbent assay

HBV: Hepatitis B Virus

HBs: Hebatitis B surface antitigen

IU: International Unit

IQR: Interquartile range

OR: Odd ratio

TT: Tetanus toxoid

Declaration

Ethical approval and consent to participate

The ethical clearance to carry out the study was approved by the joint Catholic University of Health and Allied Sciences/Bugando Medical Centre (CUHAS-BMC) research ethics and review committee (CREC) with ethical clearance number CREC/118/2016. All the parents/guardians were asked to sign informed consent on behalf of their children prior enrollment to the study.

\section{Consent for publication}

Not applicable

\section{Availability of data and material}

All data were included in this manuscript. The raw data is available upon request to the Director of research and Innovation of the Catholic University of Health and allied Sciences.

Competing of interests

No conflict of interest to declare.

Funding

This research was supported by research grant from CUHAS and ELISA KITS from SIEMENS, Enzygnost ${ }^{\circledR}$ Anti-HBs II, Germany.

\section{Authors' contributions}

DRM, SK, RR, EK, TM, MMM and SEM participated in the design of the study. EM and DRM participated in the collection of specimens and data. MMM, SEM and EM performed serological tests. SEM and $\mathrm{MMM}$ analyzed and interpreted the data. DRM wrote the first draft of the manuscript. SEM and MMM provided a critical review of the manuscript. All authors read and approved the final version of the manuscript.

\section{Acknowledgements}

The authors would like to acknowledge the support provided by Mr. Seif Abdu and all staff of the Bugando medical centre laboratory, Department of Micriobiology and Immunology-CUHAS, Makongoro reproductive and child health clinic, Mrs. Neema B. Mshana and SIMENS, Germany.

\section{References Références Referencias}

1. Lavanchy D: Hepatitis $B$ virus epidemiology, disease burden, treatment, and current and emerging 
prevention and control measures. Journal of viral hepatitis 2004, 11(2):97-107.

2. Hyams KC: Risks of chronicity following acute hepatitis $B$ virus infection: a review. Clinical Infectious Diseases 1995, 20(4):992-1000.

3. Robinson W: Hepatitis B viruses. General features (human). Encyclopedia of Virology London, Academic Press Ltd 1994:554-569.

4. Banatvala J, Van Damme P, Oehen S: Lifelong protection against hepatitis $B$ : the role of vaccine immunogenicity in immune memory. Vaccine 2000, 19(7):877-885.

5. Kwon SY, Lee CH: Epidemiology and prevention of hepatitis $B$ virus infection. The Korean journal of hepatology 2011, 17(2):87.

6. WHO: Hepatitis B facts sheet N 204. In.; 2014.

7. Poorolajal J, Mahmoodi M, Majdzadeh R, NasseriMoghaddam S, Haghdoost A, Fotouhi A: Long-term protection provided by hepatitis $B$ vaccine and need for booster dose: a meta-analysis. Vaccine 2010, 28(3):623-631.

8. Metodi J, Aboud S, Mpembeni R, Munubhi E: Immunity to hepatitis $B$ vaccine in Tanzanian under-5 children. Annals of tropical paediatrics 2010, 30(2):129-136.

9. CDC: Immunization of health-care personnel: recommendations of the Advisory Committee on Immunization Practices (ACIP). . In.; 2011.

10. Gomber S, Sharma R, Ramachandran V, Talwar V, Singh B: Immunogenicity of hepatitis $B$ vaccine incorporated into the expanded program of immunization schedule. Indian pediatrics 2000, 37(4):411-413.

11. Gowin E, Wysocki J, Kałużna E, Świątek-Kościelna B, Wysocka-Leszczyńska J, Michalak M, Januszkiewicz-Lewandowska D: Does vaccination ensure protection? Assessing diphtheria and tetanus antibody levels in a population of healthy children: A cross-sectional study. Medicine 2016, 95(49).

12. Fadugba OO, Wang L, Chen Q, Halasa NB: Immune responses to pertussis antigens in infants and toddlers after immunization with multicomponent acellular pertussis vaccine. Clinical and Vaccine Immunology 2014, 21(12):1613-1619.

13. Ball LK, Falk LA, Horne AD, Finn TM: Evaluating the immune response to combination vaccines. Clinical infectious diseases 2001, 33(Supplement 4):S299S305.

14. Rezaei M, Nooripoor S, Ghorbani R, Ramezanshams F, Mamishi S, Mahmoudi S: Seroprotection after hepatitis $B$ vaccination in children aged 1 to 15 years in central province of Iran, Semnan. Journal of preventive medicine and hygiene 2014, 55(1):1.

15. Kiteleja K: Annual implementation report 2016 Mwanza region. In. Edited by (IVD) lavd: MOHCDGEC; 2016: 14.
16. Jafarzadeh A, SAJADI S: PERSISTENCE OF ANTIHBS ANTIBODIES IN HEALTHY IRANIAN CHILDREN VACCINATED WITH RECOMBINANTHEPATITIS B VACCINE AND RESPONSE TO A BOOSTER DOSE. 2005.

17. Hsu H-M, Lee S-C, Wang M-C, Lin S-F, Chen D-S: Efficacy of a mass hepatitis $B$ immunization program after switching to recombinant hepatitis $B$ vaccine: a population-based study in Taiwan. Vaccine 2001, 19(20):2825-2829.

18. Karaoglu L, Pehlivan E, Gunes G, Genc M, Tekerekoglu S, Ercan C, Egri M, Yologlu S: Evaluation of the immune response to hepatitis $B$ vaccination in children aged 1-3 years in Malatya, Turkey. The new microbiologica 2003, 26 (4):311319.

19. WHO: Hepatitis B position paper. In.: Weekly Epidemiological Records; 2017.

20. Aghakhani A, Banifazl M, Izadi N, McFarland W, Sofian M, Khadem-Sadegh A, Pournasiri Z, Foroughi M, Eslamifar A, Ramezani A: Persistence of antibody to hepatitis $B$ surface antigen among vaccinated children in a low hepatitis $B$ virus endemic area. World Journal of Pediatrics 2011, 7 (4):358-360.

21. Whittle $H$, Jaffar $S$, Wansbrough $M$, Mendy $M$, Dumpis U, Collinson A, Hall A: Observational study of vaccine efficacy 14 years after trial of hepatitis $B$ vaccination in Gambian children. Bmj 2002, 325(7364):569.

22. But DY-K, Lai C-L, Lim W-L, Fung J, Wong DK-H, Yuen M-F: Twenty-two years follow-up of a prospective randomized trial of hepatitis $B$ vaccines without booster dose in children. Vaccine 2008, 26(51):6587-6591.

23. AlFaleh $F$, AlShehri $S$, AlAnsari $S$, AlJeffri $M$, AlMazrou Y, Shaffi A, Abdo AA: Long-term protection of hepatitis $B$ vaccine 18 years after vaccination. Journal of Infection 2008, 57(5):404-409.

24. WHO: Hepatitis B vaccines. In. Geneva; 1999.

25. Kuhail S, El Khodary R, Ahmed F: Evaluation of the routine hepatitis $B$ immunization programme in Palestine, 1996. 2000.

26. Dahifar $\mathrm{H}$ : Immunogenicity of Cuban hepatitis $B$ vaccine in Iranian children. Arch Iranian Med 2004, 7(2):89-92.

27. Goncalves L, Albarran B, Salmen S, Borges L, Fields $\mathrm{H}$, Montes $\mathrm{H}$, Soyano A, Diaz $\mathrm{Y}$, Berrueta L: The nonresponse to hepatitis $B$ vaccination is associated with impaired lymphocyte activation. Virology 2004, 326(1):20-28.

28. Kardar G, Jeddi-Tehrani M, Shokri F: Diminished Th1 and Th2 cytokine production in healthy adult nonresponders to recombinant hepatitis $B$ vaccine. Scandinavian journal of immunology 2002 , 55(3):311-314. 
29. Roome AJ, Walsh SJ, Cartter ML, Hadler JL: Hepatitis $B$ vaccine responsiveness in Connecticut public safety personnel. Jama 1993, 270(24):29312934.

30. Li H, Li R-C, Liao S-S, Yang J-Y, Zeng X-J, Wang S$\mathrm{S}$ : Persistence of hepatitis $B$ vaccine immune protection and response to hepatitis $B$ booster immunization. World journal of gastroenterology 1998, 4(6):493.

31. Fujisawa $T$, Onoue $M$, Inui $A$, Kosugi $T$ : Serial changes in titers of antibody to hepatitis $B$ surface antigen after immunization of infants born to mothers with hepatitis $B$ e antigen. Journal of pediatric gastroenterology and nutrition 1996, 23(3):270-274.

32. Lin Y-C, Chang M-H, Ni Y-H, Hsu H-Y, Chen D-S: Long-term immunogenicity and efficacy of universal hepatitis $B$ virus vaccination in Taiwan. The Journal of infectious diseases 2003, 187(1):134-138.

33. Leuridan E, Van Damme P: Hepatitis $B$ and the need for a booster dose. Clinical Infectious Diseases 2011, 53(1):68-75.

34. Simons BC, Spradling PR, Bruden DJ, Zanis C, Case S, Choromanski TL, Apodaca M, Brogdon HD, Dwyer G, Snowball M: A longitudinal hepatitis $B$ vaccine cohort demonstrates long-lasting hepatitis $B$ virus (HBV) cellular immunity despite loss of antibody against HBV surface antigen. The Journal of infectious diseases 2016, 214(2):273-280.

35. Sallam TA, Alghshm HM, Ablohom AA, Alarosi MS, Almotawakel RE, Farea $\mathrm{NH}$, Mosleh AA: Immune response to Hepatitis $B$ vaccine among children in Yemen. Saudi medical journal 2005, 26(2):281-284. 